\title{
Atividade física de lazer e uso de substâncias lícitas em uma amostra populacional de adultos jovens
}

\author{
Leisure-time physical activity and licit substance use \\ in a population sample of young adults
}

Roberta Lacerda Nasser ${ }^{1}$

Jerônimo Costa Branco ${ }^{2}$

Diogo Rizzato Lara ${ }^{3}$

Fabrício Boscolo Del Vecchio ${ }^{4}$

Carolina Wiener ${ }^{1}$

Paula Mesquita ${ }^{1}$

Luciano Dias de Mattos Souza ${ }^{1}$

Ricardo Azevedo da Silva ${ }^{1}$

Karen Jansen ${ }^{1}$

${ }^{1}$ Centro de Ciências da Vida e da Saúde, Universidade Católica de Pelotas. R. Félix da Cunha 412, Centro. 96010-000 Pelotas RS Brasil.karen.jansen@ pq.cnpq.br ${ }^{2}$ Curso de Fisioterapia, Centro Universitário

Franciscano.

${ }^{3}$ Programa de PósGraduação em Biologia Celular e Molecular, Pontifícia Universidade Católica do Rio Grande do Sul.

${ }^{4}$ Escola Superior de Educação Física, Universidade Federal de Pelotas.
Abstract The scope of this study was to assess the prevalence and factors associated with leisure-time physical activity, as well as to verify its association with licit substance use in a population sample of young adults. It is a cross-sectional population-based study with young adults aged between 18 and 35 years old, living in the urban area of the city of Pelotas in the Brazilian State of Rio Grande do Sul. Sample selection was performed by clusters, being composed of 1953 individuals, among the 82 selected census tracts. The practice of physical activity was evaluated using the International Physical Activity Questionnaire (IPAQ). The chi-square and Poisson regression tests were used for statistical analysis. The prevalence of young adults active in leisure-time physical activity was $25.3 \%$; most were single males between 18 and 23 years, belonging to the people in the highest economic bracket and not smoking on a daily basis. Specific strategies should be developed to promote an active and healthy lifestyle, focusing on incentives for older, lower income class women who smoke.

Key words Physical activity, Leisure-time activities, Tobacco, Alcohol, Young adults
Resumo O objetivo do estudo foi mensurar a prevalência e os fatores associados à prática de atividade física de lazer, bem como verificar a associação desta com o uso de substâncias lícitas em uma amostra populacional de adultos jovens. Estudo transversal de base populacional com adultos jovens de 18 a 35 anos de idade, residentes da zona urbana da cidade de Pelotas-RS (Brasil). A seleção amostral foi realizada por conglomerados, sendo constituída por 1953 indivíduos, dentre 82 setores censitários sorteados. A prática de atividade física foi avaliada por meio do Questionário Internacional de Atividade Física (IPAQ). Para a análise estatística utilizou-se a regressão de Poisson. A prevalência de jovens ativos em atividade física de lazer foi de 25,3\% e esteve associada a ser do sexo masculino, ter entre 18 e 23 anos de idade, pertencer ao maior tercil do indicador econômico nacional e não fazer uso diário de tabaco. Sabendo-se da importância da atividade física de lazer para a saúde, os incentivos para esta prática devem ser focados nos grupos de mulheres, de maior idade, de estratos econômicos inferiores e que fazem uso de tabaco.

Palavras-chave Atividade física, Atividades de lazer, Tabaco, Álcool, Adultos jovens 


\section{Introdução}

Qualquer movimento corporal produzido pelo músculo esquelético que resulta em aumento do dispêndio energético é definido como atividade física. Esta definição considera quatro contextos principais: o trabalho, as atividades domésticas, o transporte, e as atividades de lazer. Esta última inclui exercícios físicos, danças, esportes e práticas recreativas ${ }^{1}$.

A atividade física de lazer é relevante para toda população, principalmente para os jovens, pois promove diversos benefícios para a saúde, podendo contribuir para o aumento do nível de atividade física na idade adulta ${ }^{2}$. No Brasil, mais de $60 \%$ dos adultos que vivem em áreas urbanas não atingem níveis suficientes de atividade físi$\mathrm{Ca}^{2,3}$. Adicionalmente, considera-se ativo, o indivíduo que pratica no mínimo 150 minutos de atividade física por semana segundo recomendações do American College of Sport Medicine ${ }^{4}$.

A tendência à diminuição da atividade física nos dias atuais é ocasionada pela automação e modificações no ambiente de trabalho, assim como pelo decréscimo do tempo destinado ao lazer $^{5,6}$. Outro fator relacionado à diminuição da prática de atividade física é a falta de locais públicos seguros e adequados para a prática de atividades de lazer?

A inatividade física gera preocupação por parte dos órgãos de saúde pública no Brasil, pois representa importante fator de risco no desenvolvimento de doenças cardiovasculares, osteoporose, hipertensão arterial, diabetes mellitus II e alguns tipos de câncer ${ }^{3}$. Além destes riscos à saúde, este comportamento pode ser ainda mais prejudicial quando associado a outros fatores de risco comuns, como o uso de tabaco e de bebidas alcoólicas ${ }^{8}$.

Neste contexto, a maioria dos estudos mostram que jovens fumantes e que utilizam bebidas alcoólicas apresentam menores níveis de atividade física ${ }^{9,10}$. Além disso, a prática de atividades físicas de lazer diminui consideravelmente com aumento da idade, principalmente da adolescência para a idade adulta ${ }^{11}$

Sendo assim, o objetivo do presente estudo foi mensurar a prevalência e os fatores associados à prática de atividade física de lazer, bem como verificar a associação desta com o uso de substâncias lícitas em uma amostra populacional de adultos jovens de uma cidade do Sul do Brasil.

\section{Método}

Estudo transversal de base populacional com adultos jovens residentes na zona urbana da cidade de Pelotas-RS (Brasil). A seleção da amostra foi realizada por meio de conglomerados considerando os dados fornecidos pelo Instituto Brasileiro de Geografia e Estatística (IBGE), sendo que a divisão censitária atual é de 495 setores na zona urbana e a população é de, aproximadamente, 97 mil adultos na faixa etária de 18 a 35 anos. A fim de garantir a aleatoriedade da amostra, os setores foram ordenados por localização e sistematicamente selecionados a cada seis, resultando em 82 censitários. Dentre os setores selecionados, deuse início a identificação da amostra a partir do ponto de referência estabelecido pelo IBGE, com intercepto de quatro domicílios a cada sorteado.

O cálculo de tamanho amostral para verificar a prevalência de atividade física de lazer foi estimado considerando a população de 97 mil adultos jovens, prevalência de atividade física de lazer de $27 \%$, erro esperado de até $3 \%$ e nível de confiança de $95 \%$, para tal, 834 sujeitos seriam necessários (dados obtidos do estudo piloto). Não obstante, um n amostral de 1070 adultos jovens foi necessário para verificar associações com razão de prevalência de 1,3 entre expostos e não expostos, com nível de confiança de $95 \%$, poder de $80 \%$ e prevalência de $27 \%$ de atividade física de lazer. Acrescentando-se 20\% para possíveis fatores de confusão, o n mínimo necessário passou para 1284 sujeitos.

Foram considerados elegíveis adultos jovens de ambos os sexos da cidade de Pelotas-RS. Como critério de inclusão, assumiu-se ter de 18 a 35 anos de idade, residir na zona urbana da cidade, no domicílio sorteado, aceitar participar espontaneamente da investigação e assinar o termo de consentimento livre e esclarecido. Aqueles com incapacidade de responderem à entrevista por problemas físicos e/ou cognitivos foram excluídos da amostra.

Neste estudo foram consideradas as seguintes variáveis independentes: sexo, cor da pele, idade, situação conjugal, trabalho, indicador econômico e uso de tabaco e álcool. O desfecho foi a prática de atividade física de lazer (prática de uma modalidade de esporte/exercício físico descrevendo sua intensidade pelo tipo, bem como, sua frequência semanal e duração diária).

As variáveis demográficas foram coletadas com questionários devidamente estruturados para esta finalidade. Para registro do nível econômico, empregou-se o Indicador Econômico Na- 
cional (IEN) ${ }^{12}$, classificado por tercis, de acordo com a distribuição na amostra estudada. Este instrumento foi desenvolvido a partir de 12 bens de consumo e da escolaridade do chefe da família, sendo baseado no Censo Demográfico Brasileiro de 2000 do IBGE.

O uso de tabaco foi considerado quando o sujeito respondeu fazer uso de pelo menos um cigarro/dia na última semana; o abuso de álcool foi considerado quando o jovem respondeu positivo para pelo menos duas questões do instrumento de rastreamento de uso problemático de álcool, conhecido pelo acrônimo referente às suas quatro perguntas - Cut down, Annoyed by criticism, Guilty e Eye-opener (CAGE) ${ }^{13}$.

Para avaliar a prática de atividade física habitual, os jovens responderam ao International Physical Activity Questionnaire (IPAQ) ${ }^{14}$ em sua versão curta. Neste estudo foram considerados ativos os indivíduos que relataram praticar 150 minutos por semana ou mais, os demais compuseram o grupo definido como de inativos ${ }^{4}$. Instrumento que é condizente com outras publicações da área e proposto pela Organização Mundial de Saúde, validado em âmbito internacional e nacional, que permite avaliar a prática de atividade física em estudos populacionais de diferentes países e contextos socioculturais ${ }^{14}$.

Os entrevistadores eram acadêmicos dos cursos de Fisioterapia, Medicina e Psicologia do Centro de Ciências da Vida e da Saúde da Universidade Católica de Pelotas (UCPel), bolsistas de iniciação científica, que foram devidamente treinados para a aplicação dos instrumentos e participaram do estudo piloto, realizado em dois setores censitários que não foram considerados na amostra final. A coleta de dados foi realizada com netbooks para a inclusão simultânea dos dados no programa Epi-Info 6.04d.

Para a análise dos dados foi utilizado o programa Stata 11. Inicialmente, os dados foram descritos por frequência absoluta e relativa ( $\mathrm{Ta}$ bela 1). Para a análise bruta dos dados foi utilizado o teste Qui-Quadrado a fim de verificar a associação entre as variáveis independentes e o desfecho (Tabela 2).

A análise ajustada dos fatores associados à prática de atividade física de lazer foi realizada por regressão de Poisson (Tabela 3). Neste modelo de análise, as variáveis independentes com p-valor $<0,20$ foram divididas em dois níveis hierárquicos, no qual o primeiro foi composto pelas variáveis sociodemográficas (sexo, idade, situação conjugal, escolaridade e indicador econômico) e o segundo pelas variáveis comporta- mentais (uso diário de tabaco e abuso de álcool). Foram consideradas diferenças estatisticamente significativas quando $\mathrm{p}<0,05$, com $\mathrm{p}$-valor do teste de linearidade quando variável independente ordinal.

Este estudo faz parte de um projeto maior, financiado pelo edital do Programa de Apoio a Núcleos de Excelência (PRONEX) e da Fundação de Amparo à Pesquisa do Estado do Rio Grande do Sul (FAPERGS), que teve aprovação pelo Comitê de Ética da Universidade Católica de Pelotas.

Tabela 1. Características da amostra.

\begin{tabular}{|c|c|}
\hline Variáveis & $\begin{array}{c}\text { Distribuição } \\
\text { da amostra }\end{array}$ \\
\hline \multicolumn{2}{|l|}{ Sexo } \\
\hline Feminino & $1073(54,9)$ \\
\hline Masculino & $880(45,1)$ \\
\hline \multicolumn{2}{|l|}{ Cor da pele } \\
\hline Branco & $1483(75,9)$ \\
\hline Não branco & $470(24,1)$ \\
\hline \multicolumn{2}{|l|}{ Idade } \\
\hline De 18 a 23 anos & $762(39,1)$ \\
\hline De 24 a 29 anos & $622(31,8)$ \\
\hline De 30 a 35 anos & $569(29,1)$ \\
\hline \multicolumn{2}{|l|}{ Situação conjugal } \\
\hline Solteiro/ divorciado & $1230(63,1)$ \\
\hline Casado/ vive com companheiro & $720(36,9)$ \\
\hline \multicolumn{2}{|l|}{ Trabalho atual } \\
\hline Não & $794(40,7)$ \\
\hline Sim & $1158(59,3)$ \\
\hline \multicolumn{2}{|l|}{ Escolaridade } \\
\hline $\begin{array}{l}\text { Ensino Fundamental ou Médio } \\
\text { incompleto }\end{array}$ & $597(30,6)$ \\
\hline Ensino Médio completo ou Superior & $1352(69,4)$ \\
\hline \multicolumn{2}{|l|}{ Indicador econômico nacional } \\
\hline 1 (tercil mais baixo) & $648(33,3)$ \\
\hline 2 & $655(33,7)$ \\
\hline 3 & $641(33,0)$ \\
\hline \multicolumn{2}{|l|}{ Uso diário de tabaco } \\
\hline Não & $1521(78,3)$ \\
\hline Sim & $421(21,7)$ \\
\hline \multicolumn{2}{|l|}{ Abuso de álcool $(\mathrm{CAGE} \geq 2)^{*}$} \\
\hline Não & $1754(90,4)$ \\
\hline Sim & $187(9,6)$ \\
\hline Total & $1953(100)$ \\
\hline
\end{tabular}

"Variável com 1 missing. 


\section{Resultados}

A amostra do estudo foi composta por 1953 indivíduos de 18 a 35 anos de idade. Dentre a amostra total, a maior proporção foi de mulheres (54,9\%), sujeitos de cor da pele branca $(75,9 \%)$, jovens sem companheiro(a) $(63,1 \%)$ e que possuíam ensino médio completo ou superior $(69,4 \%)$. A idade dos jovens e o IEN foram apresentados em tercis. Em relação ao uso de substâncias psicoativas, $21,7 \%$ da amostra referiu fazer uso diário de tabaco e $9,6 \%$ apontou para abuso de bebida alcoólica (Tabela 1).

Foram considerados ativos para prática habitual de atividade física de lazer 25,3\% $(\mathrm{n}=490)$ dos jovens, sendo $17,7 \%$ entre as mulheres e $34,5 \%$ entre os homens (Tabela 2 ).
$\mathrm{Na}$ análise bruta dos dados, esteve associado à prática de atividade física de lazer entre adultos jovens: ser do sexo masculino $(\mathrm{p}<0,001)$, ter idade entre 18 e 23 anos $(p<0,001)$, ser solteiro/divorciado $(\mathrm{p}=0,001)$, possuir ensino médio completo ou superior $(\mathrm{p}<0,001)$, apresentar o maior tercil do indicador econômico nacional ( $\mathrm{p}$ $<0,001)$ e não fazer uso diário de tabaco ( $\mathrm{p}<$ 0,001) (Tabela 2).

Após a análise ajustada dos dados, permaneceu associado à prática habitual de atividade física de lazer: ser do sexo masculino (RP 1,79; IC95\% 1,52 - 2,10); ter entre 18 e 23 anos de idade (RP 1,57; IC95\% 1,26-1,96); pertencer ao indicador econômico mais alto (RP 1,46; IC95\% 1,19 - 1,79); e não fazer uso diário de tabaco (RP 1,59; IC95\% 1,26 - 2,02) (Tabela 3).

Tabela 2. Fatores associados à prática de atividade física de lazer entre adultos jovens, análise bruta dos dados por teste Qui-quadrado.

\begin{tabular}{|c|c|c|c|}
\hline Variáveis & $\begin{array}{c}\text { Atividade } \\
\text { Física de Lazer }\end{array}$ & $\begin{array}{l}\text { Razão de prevalência } \\
\text { bruta (IC } 95 \%)\end{array}$ & P-valor \\
\hline Sexo & & & $<0,001$ \\
\hline Feminino & $189(17,7)$ & 1,00 & \\
\hline Masculino & $301(34,5)$ & $1,02(0,87-1.20)$ & \\
\hline Cor da pele & & & 0,826 \\
\hline Branco & $370(25,1)$ & 1,00 & \\
\hline Não branco & $120(25,8)$ & $1,02(0,85-1,22)$ & \\
\hline Idade $^{*}$ & & & $<0,001$ \\
\hline De 18 a 23 anos & $234(31,0)$ & $1,76(1,43-2,16)$ & \\
\hline De 24 a 29 anos & $156(25,2)$ & $1,43(1,14-1,79)$ & \\
\hline De 30 a 35 anos & $100(17,6)$ & 1,00 & \\
\hline Situação conjugal & & & 0,001 \\
\hline Solteiro/ divorciado & $350(28,6)$ & $1,46(1,22-1,73)$ & \\
\hline Casado/ Vive com companheiro & $140(19,6)$ & 1,00 & \\
\hline Trabalho atual & & & 0,110 \\
\hline Não & $184(23,3)$ & 1,00 & \\
\hline Sim & $306(26,6)$ & $1,14(0,97-1,33)$ & \\
\hline Escolaridade & & & $<0,001$ \\
\hline Ensino Fundamental ou Médio incompleto & $118(19,9)$ & 1,00 & \\
\hline Ensino Médio completo ou Superior & $369(27,4)$ & $1,37(1,14-1,65)$ & \\
\hline Indicador econômico nacional ${ }^{*}$ & & & $<0,001$ \\
\hline 1 (tercil mais baixo) & $144(33,6)$ & $1,78(1,46-2,16)$ & \\
\hline 2 & $114(26,1)$ & $1,26(1,02-1,56)$ & \\
\hline 3 & $95(19,3)$ & 1,00 & \\
\hline Uso diário de tabaco & & & $<0,001$ \\
\hline Não & $421(27,9)$ & $1,74(1,37-2,20)$ & \\
\hline Sim & $67(16,0)$ & 1,00 & \\
\hline Abuso de álcool (CAGE $\geq 2)$ & & & 0,135 \\
\hline Não & $432(24,8)$ & 1,00 & \\
\hline Sim & $56(30,1)$ & $1,21(0,96-1,53)$ & \\
\hline Total & $490(25,3)$ & -- & --- \\
\hline
\end{tabular}

${ }^{*}$ P-valor com teste de linearidade. 
Tabela 3. Fatores associados à prática de atividade física de lazer entre adultos jovens, análise ajustada dos dados por regressão de Poisson.

\begin{tabular}{|c|c|c|}
\hline Variáveis & Razão de prevalência ajustada (IC 95\%) & P-valor \\
\hline Sexo & & $<0,001$ \\
\hline Feminino & 1,00 & \\
\hline Masculino & $1,79(1,52-2,10)$ & \\
\hline Idade $^{*}$ & & $<0,001$ \\
\hline De 18 a 23 anos & $1,57(1,26-1,96)$ & \\
\hline De 24 a 28 anos & $1,39(1,11-1,74)$ & \\
\hline De 29 a 35 anos & 1,00 & \\
\hline Situação conjugal & & 0,198 \\
\hline Solteiro/ divorciado & $1,13(0,94-1,37)$ & \\
\hline Casado/ vive com companheiro & 1,00 & \\
\hline Escolaridade & & 0,346 \\
\hline Ensino Fundamental ou Médio incompleto & 1,00 & \\
\hline Ensino Médio completo ou Superior & $1,09(0,90-1,33)$ & \\
\hline Indicador econômico nacional $^{*}$ & & $<0,001$ \\
\hline 1 (tercil mais baixo) & 1,00 & \\
\hline 2 & $1,14(0,92-1,41)$ & \\
\hline 3 & $1,46(1,19-1,79)$ & \\
\hline Uso diário de tabaco & & $<0,001$ \\
\hline Não & $1,59(1,26-2,02)$ & \\
\hline Sim & 1,00 & \\
\hline Abuso de álcool $(\mathrm{CAGE} \geq 2)^{*}$ & & 0,317 \\
\hline Não & 1,00 & \\
\hline Sim & $1,12(0,88-1,41)$ & \\
\hline
\end{tabular}

P-valor com teste de linearidade.

\section{Discussão}

A presente investigação objetivou mensurar a prevalência e os fatores associados à prática de atividade física de lazer em uma amostra populacional de adultos jovens. Sendo assim, para esta amostra, 25,3\% dos entrevistados foram considerados ativos para prática habitual de atividade física de lazer, resultados similares a outros estudos que verificaram baixa prevalência de atividade física no tempo destinado ao lazer ${ }^{15-17}$. Além disso, foi possível verificar que indivíduos do sexo masculino, com idades entre 18 e 23 anos, de maior nível educacional e maior classificação quanto ao indicador econômico nacional, bem como, aqueles não faziam uso diário de tabaco apresentaram maior prevalência de atividade física de lazer. Com efeito, os dados da presente investigação corroboram outros estudos ${ }^{18,19}$.

Em relação à prática de atividade física de lazer entre homens e mulheres, observa-se uma tendência de as mulheres praticarem menos quando comparadas aos homens ${ }^{20,21}$. Autores justificam que a prevalência de atividade física é maior entre os homens, porque além de atividades como caminhadas, eles também realizam esportes coletivos com maior frequência ${ }^{22}$. Além disso, as mulheres procuram a prática de atividade física por motivos estéticos, a qual acaba restringindo-se a atividades individuais e monótonas o que aumenta a probabilidade de desistências ${ }^{22,23}$.

Quanto à faixa etária de maior prevalência de atividade física de lazer, os achados têm sido consistentemente associados à juventude ${ }^{19}$, sendo que estudo multicêntrico, realizado pelo Ministério da Saúde com pessoas de 18 anos ou mais, encontrou maior prevalência de atividade física de lazer entre indivíduos de 18 a 24 anos de idade ${ }^{18}$.

Autores têm evidenciado que crianças e adolescentes ativos, apresentam maiores chances de se tornarem adultos ativos ${ }^{24}$. As atividades esportivas escolares promovem habilidades e diversão ajudando na fundamentação de atividades físicas na vida adulta, e consequentemente diminuindo 
o risco de doenças. Por isso, se torna importante políticas que incentivem as atividades esportivas nesses grupos etários mais jovens ${ }^{25}$.

Em estudos realizados para verificar a associação da atividade física de lazer com a escolaridade, foram encontrados resultados similares ao deste estudo, sendo que, em geral, quanto maior a escolaridade do indivíduo, maior a probabilidade de praticar atividades físicas no seu tempo destinado ao lazer ${ }^{26,27}$. A diminuição da atividade física de lazer por pessoas com menor escolaridade pode ser explicada pela inserção em jornadas laborais extensas, com atividades pesadas e extenuantes, o que possivelmente não estimula essa prática $^{28}$. Na amostra deste estudo, pessoas com maior renda familiar praticaram mais atividades físicas de lazer. Em geral, estudos têm indicado que quanto maior o poder aquisitivo de um indivíduo, maior será a probabilidade do mesmo praticar atividades físicas no seu tempo destinado ao lazer $^{16,21,27,29}$. Não obstante, sujeitos que compõem um quadro de maiores recursos financeiros e maior instrução têm mais acesso e preocupação com a promoção e prevenção em saúde ${ }^{30}$.

Observou-se, também, que pessoas sem companheiro(a) apresentaram maior prevalência de prática de atividade física de lazer antes do ajuste para variáveis sociodemográficas. Este resultado é muito parecido com os encontrados em outros estudos, em que pessoas solteiras relataram maior envolvimento em atividades físicas durante as horas de lazer ${ }^{26,28}$. No entanto, vale dizer que já foi encontrado resultado diferente quanto à situação conjugal, com ausência de associação desta com a prática de atividade física de lazer ${ }^{21}$. Uma explicação para maior prevalência de pessoas solteiras serem fisicamente mais ativas pode decorrer da possibilidade de haver mais tempo disponível para a prática de atividades físicas, visto que a união conjugal faz com que a pessoa dedique mais tempo em família ${ }^{16}$.

Estudos realizados com trabalhadores ${ }^{17} \mathrm{e}$ com estudantes adolescentes ${ }^{31}$ têm indicado que quantidades superiores de exercícios fisicos estão associadas a níveis mais baixos de cigarro e maco$\mathrm{nha}^{17,31}$. Tais achados se assemelham aos nossos, de que os jovens ativos em atividades de lazer em sua maioria não são fumantes. $\mathrm{Na}$ adolescência, a prática regular de exercícios físicos interfere no início do tabagismo e é considerada fator protetor contra o início deste hábito. Além disto, a atividade física pode colaborar para o abandono do hábito de fumar quando este estiver estabelecido ${ }^{9,32,33}$.
Outro fato relevante é a relação entre tabaco e aptidão física, fumantes apresentam menor resistência aeróbica em função da sobrecarga causada pelo cigarro no sistema cardiorrespiratório ${ }^{34,35}$, o que pode propiciar o abandono da prática de atividade física em função de uma baixa aptidão física. Mas a associação entre a prática de atividade física e o tabagismo está limitada ao viés de causalidade, podendo a atividade física ser vista como um fator de proteção para o habito de fumar ou o fato de ser tabagista consequentemente levar à inatividade física.

A Relação do álcool com a prática de atividade física parece ser conflitante ${ }^{10,36,37}$. Ao mesmo tempo em que encontramos em nosso estudo uma maior frequência de abusadores de álcool entre os indivíduos ativos, apesar desta associação não ser estatisticamente significativa; estudos têm demonstrado que a prática de atividade física de lazer está relacionada com um maior consumo de álcool ${ }^{17,38}$. Por outro lado, pesquisadores indicam que a prática de atividade física, devido ao aumento dos processos de autorreparos endógenos, possa servir para o tratamento do consumo do álcool ${ }^{39}$; em estudo com animais, o exercício é responsável por reverter danos ao cérebro provocados pelo excesso de álcool ${ }^{40}$; e em estudo com atletas que realizaram alguns testes de aptidão física, com e sem a presença de álcool na corrente sanguínea, foi encontrado pior resultado entre os que haviam feito uso dessa substância no dia anterior aos testes físicos ${ }^{41}$.

Considerando os achados deste trabalho e os estudos disponíveis na literatura, temos como hipótese que algumas características desta amostra de fisicamente ativos se assemelham às características daqueles que fazem uso abusivo de álcool, como ser jovem, do sexo masculino e ter períodos de lazer. Desta forma, a prevalência de abuso de álcool é maior entre os ativos, porém sem poder estatístico para confirmar a associação. Em virtude das informações citadas acima, estudos longitudinais e experimentais com poder adequado são necessários para confirmar a relação e os efeitos da prática de atividade física entre os indivíduos com transtornos de abuso de álcool, para apontar os potenciais mecanismos de ação envolvidos.

Sabendo-se da importância da atividade física de lazer para a saúde, os incentivos para esta prática devem ser focados nos grupos de mulheres, de maior idade, de estratos econômicos inferiores e que fazem uso de tabaco. Principalmente 
pelo fato de nossos resultados evidenciarem que a prática habitual de atividade física de lazer esta associada ao sexo masculino, entre aqueles com menos idade, pertencer ao indicador econômico mais alto e não fazer uso diário de tabaco.

\section{Colaboradores}

\section{Referências}

RL Nasser, JC Branco, C Wiener, P Mesquita e K Jansen contribuíram na concepção do projeto, coleta e análise dos dados, redação e discussão do artigo; FB Del Vecchio realizou uma revisão minuciosa do artigo e aprovou a versão final; RA Silva, LDM Souza e DR Lara contribuíram na concepção do projeto, realizaram uma revisão minuciosa do artigo e aprovaram a versão final do artigo.

1. Caspersen CJ, Powell KE, Christenson GM. Physical activity, exercise, and physical fitness: definitions and distinctions for health-related research. Public Health Reports 1985; 100(2):126-31.

2. Hallal PC, Victora CG, Wells JC, Lima RC. Physical inactivity: prevalence and associated variables in Brazilian adults. Med Sci Sports Exerc 2003; 35(11):18941900.

3. Brasil. Ministério da Saúde. Programa Nacional de Promoção da Atividade Física "Agita Brasil": atividade física e sua contribuição para a qualidade de vida. Rev Saude Publica 2002; 36(2):254-256.

4. Haskell WL, Lee IM, Pate RR, Powell KE, Blair SN, Franklin BA, Macera CA, Heath GW, Thompson PD, Bauman A. Physical activity and public health: updated recommendation for adults from the American College of Sports Medicine and the American Heart Association. Med Sci Sports Exerc 2007; 39(8):1423-1434.

5. Powell KE, Paffenbarger RS. Work- shop on epidemiologic and public health aspects of physical activity and exercise: A summary. Public Health Rep 1985; 100(2):111-126.

6. Owen N, Healy GN, Matthews CE, Dunstan DW. Too much sitting: the population health science of sedentary behavior. Exerc and Sport Sciences Reviews 2010; 38(3):105-113.

7. Sallis JF, Conway TL, Prochaska JJ, Mckenzie TL, Marchall SJ, Brown M. The association of school environments with youth physical activity. Am J Public Health 2001; 91(4):618-620.

8. Ceschini FL, Florindo AA, Benício MHDA. Nível de atividade física em adolescentes de uma região de elevado índice de vulnerabilidade juvenil. Rev Bras Ci e Mov 2007; 15(4):67-78.

9. Higgins JW, Gaul C, Gibbons S, Van Gyn G. Factors influencing physical activity levels among Canadian youth. Can J Publ Health 2003; 94(1):45-51.

10. Singleton RA. Collegiate alcohol consumption and academic performance. J Stud Alcohol Drugs 2007; 68(4):548-555.

11. Malina RM. Physical activity and fitness: pathways from childhood to adulthood. Am J Hum Bio 2001; 13(2):162-172.

12. Barros AJD, Victoria CG. Indicador econômico para o Brasil baseado no censo demográfico de 2000. Rev Saude Publica 2005; 39(4):523-529. 
13. Mayfield D, McLeod G, Hall P. The CAGE questionnaire: validation of new alcoholism screning instrument. Am J Psychiatr 1974; 131(10):1121-1123.

14. Craig CL, Marchall Al, Sjostrom M, Baumam AE, Booth ML, Ainsworthh BE, Pratt M, Ekelund U, Yngve A, Sallis JF, Oja P. International Physical Activity Questionnaire: 12-country reability and validity. Med Sci Sports Exerc 2003; 35(8):1381-1395.

15. Knuth AG, Malta DC, DumithI SC, Pereira CA, Neto OLM, Temporão JG, Penna G, Hallal PC. Prática de atividade física e sedentarismo em brasileiros: resultados da Pesquisa Nacional por Amostra de Domicílios (PNAD) 2008. Cien Saude Colet 2011; 16(9):3697-3705

16. Mensink GBM, Loose N, Oomen C. Physical activity and its association with other lifestyle factors. Eur J Epidemiol 1997; 13(7):771-778.

17. Barros MVG, Nahas MV. Comportamentos de risco, auto-avaliação do nível de saúde e percepção de estresse entre trabalhadores da indústria. Rev Saude Publica 2001; 35(6):554-563.

18. Brasil. Ministério da Saúde (MS). Secretaria de Vigilância em Saúde. Secretaria de Gestão Estratégica e Participativa. Vigitel Brasil 2007: vigilância de fatores de risco e proteção para doenças crônicas por inquérito telefônico. Brasília: MS; 2008.

19. Bicalho PG, Hallal PC, Gazzinelli A, Knuth AG, Meléndez GV. Atividade física e fatores associados em adultos de área rural em Minas Gerais, Brasil. Rev Saude Publica 2010; 44(5):884-893.

20. Malta DC, Sardinha LMV, Mendes I, Barreto SM, Giatti L, Castro IRR, Moura L, Dias AJR, Crespo C. Prevalência de fatores de risco e proteção de doenças crônicas não transmissíveis em adolescentes: resultados da Pesquisa Nacional de Saúde do Escolar (PeNSE), Brasil, 2009. Cien Saude Colet 2010; 15(Supl. 2):3009-3019.

21. Salles-Costa R, Werneck GL, Lopes CS, Faerstein E. Associação entre fatores sociodemográficos e prática de atividade física de lazer no Estudo Pró-Saúde. Cad Saude Publica 2003; 19(4):1095-1105.

22. Azevedo MR, Araújo CLP, Reichert FF, Siqueira FV, Silva MC, Hallal PC. Gender differences in leisure-time physical activity. Int J Public Health 2007; 52(1):8-15

23. Monteiro CA, Conde WL, Matsudo SM, Matsudo VR, Bonseñor IM, Lotufo PA. A descriptive epidemiology of leisure-time physical activity in Brazil, 19961997. Rev Panam Salud Publica 2003; 14(4):246-254.

24. Azevedo MR, Araujo CL, Silva MC, Hallal PC. Tracking of physical activity from adolescence to adulthood: a population-based study. Rev Saude Publica 2007; 41(1):69-75

25. Houston TK, Meoni LA, Ford DE, Brancati FL, Cooper LA, Lê Liang KY, Klag MJ. Sports ability in young men and the incidence of cardiovascular disease. Am J Med 2002; 112(9):733-734

26. Martinez-Gonzalez MA, Varo JJ, Santos Jl, De Irala J, Gibney M, Kearney J, Martínez JA. Prevalence of physical activity during leisure time in the Europe Union. Med Sci Sports Exerc 2001; 33(7):1142-1146.

27. Troiano RP, Macera CA, Ballard-Bar-Bash R. Be physically active each day. How can we know? J Nut 2001; 131(2S-1):451-460.

28. Pitanga FJG, Lessa I. Prevalência e fatores associados ao sedentarismo no lazer em adultos. Cad Saude Publica 2005; 21(3):870-877.
29. Ford ES, Merritt RK, Heath GW, Powell KE, Wasburn RA, Kriska A, Haile G. Physical activity behaviors in lower and higher socioeconomic status populations. Am J Epidemiol 1991; 133(12):1246-1256.

30. Yu ZM, Parker L, Dummer TJ. Depressive symptoms, diet quality, physical activity, and body composition among populations in Nova Scotia, Canada: report from the Atlantic Partnership for Tomorrow's Health. Prev Med 2014; 61:106-113.

31. Yvonne M, Terry-McElrath MSA, O’Malley PhD, Lloyd $\mathrm{D}$, Johnston PhD. Exercise and substance use among American youth, 1991-2009. Am J Prev Med 2011; 40(5):530-540.

32. Holmen TL, Barrett-Connor E, Clausen J, Holmen J, Bjermer L. Physical exercise, sports, and lung function in smoking versus nonsmoking adolescents. Eur Respir J 2002; 19(1):8-15

33. Costa AA, Jansen U, Lopes AJ, Trindade FP, Maiworm AI, Salles N, Meirelles LR, Maeda TY, Noronha Filho AJ Jansen JM. Tabagismo. Ars Cvrandi 2002; 35(8):40-47.

34. Misigoj-Durakovic M, Bok D, Soric M, Dizdar D, Durakovic Z, Jukic I. The effect of cigarette smoking history on muscular and cardiorespiratory endurance. J Addict Dis 2012; 31(4):389-396.

35. Pavić I, Jurica SA, Pavić P, Bogović JC, Krmek M, Dodig S.The effects of parental smoking on anthropometric parameters, peak expiratory flow rate and physical condition in school children. Coll Antropol 2014; 38(1):189-194.

36. Ströhle A, Höfler M, Pfister H, Müller AG, Hoyer J, Wittchen HU, Lieb R. Physical activity and prevalence and incidence of mental disorders in adolescents and young adults. Psychol Med 2007; 37(11):1657-1666.

37. Dunn MS. Association between physical activity and substance use behaviors among high school students participating in the 2009 Youth Risk Behavior Survey. Psychol Rep 2014; 114(3):675-685.

38. Costa JSD, Silveira MF, Gazalle FK, Oliveira SS, Hallal PC, Menezes AMB, Gigante DP, Olinto MTA, Macedo S. Consumo abusivo de álcool e fatores associados: estudo de base populacional. Rev Saude Publica 2004; 38(2):284-291

39. Murphy TJ, Pagano RR, Marlatt GA. Lifestyle modification with heavy alcohol drinkers: effects of aerobic exercise and meditation. Addict Behav 1986; 11(2):175186.

40. Maynard ME, Leasure JL. Exercise enhances hippocampal recovery following binge ethanol exposure. PLoS One 2013; 30(9):76644.

41. Prentice C, Stannard SR, Barnes MJ. Effects of heavy episodic drinking on physical performance in club level rugby union players. J Sci Med Sport 2014; (Supl. 14):S1440-2440

Artigo apresentado em 03/09/2014

Aprovado em 23/03/2015

Versão final apresentada em 25/03/2015 